\title{
Characterization of Type I and IV Collagens by Raman Microspectroscopy: Identification of Spectral Markers of the Dermo-Epidermal Junction
}

\author{
T. T. Nguyen, ${ }^{1}$ C. Gobinet, ${ }^{1}$ J. Feru, ${ }^{2}$ S. Brassart -Pasco, ${ }^{2}$ M. Manfait, ${ }^{1}$ and O. Piot $^{1}$ \\ ${ }^{1}$ CNRS FRE 3481, MEDyC, UFR Pharmacie, Université de Reims Champagne-Ardenne, \\ 51 rue Cognacq-Jay, 51096 Reims Cedex, France \\ ${ }^{2}$ CNRS FRE 3481, MEDyC, UFR Medecine, Université de Reims Champagne-Ardenne, \\ 51 rue Cognacq-Jay, 51096 Reims Cedex, France
}

Correspondence should be addressed to O. Piot, olivier.piot@univ-reims.fr

Copyright (C) 2012 T. T. Nguyen et al. This is an open access article distributed under the Creative Commons Attribution License, which permits unrestricted use, distribution, and reproduction in any medium, provided the original work is properly cited.

\begin{abstract}
Type I and IV collagens are important constituents of the skin. Type I collagen is found in all dermal layers in high proportion, while type IV collagen is localized in the basement membrane of the dermo-epidermal junction (DEJ). These proteins are strongly altered during aging or cancer progression. Although they possess amino acid compositions which, are close, they present also important structural differences inducing specific physicochemical properties. Raman spectroscopy is based on a nondestructive interaction of the light with the matter. This technique permits to probe the intrinsic molecular composition of the samples without staining or particular preparation. The aim of our research is to study the correlation between the molecular conformations of type I and IV collagens and their Raman features. We showed that signals specific of each protein can be revealed and that they translate structural differences between the two collagens. From this collagens spectral characterization, the analysis of skin sections also permitted to identify spectral markers of dermis, epidermis, and epidermis/dermis interface. These preliminary results represent basic data for further studies, particularly to probe skin molecular alterations induced by chronologic aging.
\end{abstract}

Keywords: Skin, Raman microspectroscopy, type I collagen, type IV collagen, epidermis/dermis interface

\section{Introduction}

Collagens I and IV are keyconstituents of the skin and are involved in various physiopathological alterations associated with cancer or aging. Type I collagen is one of the most abundant proteins in mammals and is found in all dermal layers of skin in high proportion (85-90\%). It contributes to the great tensile strength and allows withstanding deformation of the dermis. In chronologic aging, type I collagen of dermis becomes sparser and less soluble [1]. In basal cell carcinoma, type I collagen is found degraded at the level of the peritumoral dermis [2]. Type IV collagen is localized in the basement 
membrane of the dermo-epidermal junction (DEJ); this constituent is crucial to maintain the integrity, the stability, and the functionality of this very thin membrane. Antiangiogenic and antitumor activities of type IV collagen were demonstrated in vitro; these properties were attributed to the NC1 domain of the protein [3-8]. In skin aging, the thickness of the basement membrane increases but the type IV collagen content declines [9].

All of the collagen types consist of a set of three polypeptide chains called tropocollagen, about $300 \mathrm{~nm}$ and $360 \mathrm{~nm}$ in length for type I and IV collagens, respectively [10]. Each polypeptide chain is characterized by a Gly-X-Y sequence where $\mathrm{X}$ corresponds frequently to proline and $\mathrm{Y}$ to hydroxyproline [11]. For each Gly-X-Y tripeptide unit, there is only one direct intrachain hydrogen bond between the $\mathrm{N}-\mathrm{H}$ group of glycine and the $\mathrm{C}=\mathrm{O}$ group of $\mathrm{X}$ residue. In addition, stabilizing interactions originate from the network of hydrogen bondings between water and hydroxyproline residues [12]. These different types of interactions ensure tropocollagen to present a triple-helical conformation. For type I collagen of the dermis, tropocollagens are associated with each other by mainly three different types of cross-links (lysinonorleucine, glucosyl-galactosyl-hydroxylysinonorleucine, and histidinohydroxymerosine links), forming a very resistant fiber [10]. So, "fibrillar" is a current adjective for type I collagen. Type IV collagen structure is different from the collagen I. It does not form fibers but a plane network, stabilized by an arrangement of N-terminal domains of four tropocollagens. These domains are associated in tetramers, through disulfide bonds and lysinonorleucine and glucosylgalactosyl-hydroxylysinonorleucine cross-links to constitute the 7S domain [10]. Another feature of collagen IV is the presence of several interruptions within the Gly-X-Y sequence [13].

Presently, immunohistochemistry and western blot techniques are the main techniques to probe collagen IV content in the basement membrane and to investigate molecular alterations such as those associated with aging $[10,14]$. The weak point of these techniques is their destructive nature and the necessity to use extrinsic labeling. In contrast, Raman spectroscopy is a nondestructive approach based on the interaction of the light with the matter. This vibrational technique is an interesting label-free tool to probe the intrinsic molecular composition of a sample and also to access some structural information by preserving the sample integrity. To our knowledge, no studies referring to the analysis of human collagen IV by Raman spectroscopy has been published up to now. For type I collagen, researches showed the advantage of Raman microspectroscopy to access in situ conformational information on skin sections $[2,15]$.

In our study, Raman spectroscopy was used to study the correlation between the molecular conformations of the type I and IV collagens and their Raman features. From skin sections, we also examined the Raman signal at the level of DEJ, compared to dermis and epidermis signatures.

\section{Materials and Methods}

\subsection{Raman Instrumentation}

Raman data were collected with a LabRAM microspectrometer (Horiba Jobin Yvon, Villeneuve d'Ascq, France), equipped with a CCD detector, and using a $660 \mathrm{~nm}$ laser diode as excitation source. A 100x objective (NA $=0.8$, Leica) permits to focus the incident laser beam and to collect the Raman scattering. The spectral resolution is $4 \mathrm{~cm}^{-1}$ using a 950 grooves $/ \mathrm{mm}$ holographic grating. 


\subsection{Reference Collagens}

Pure type IV collagen was obtained from Sigma-Aldrich. Type I collagen was prepared in our laboratory from Sprague Dawley rat (Depre, St. Doulchard, France) tail tendons by $0.5 \mathrm{M}$ acetic acid extraction [16], dialyzed against distilled water and lyophilized.

\subsection{Raman Analysis of Reference Collagens and of Tissue Sections}

Type I and IV collagens were deposited on $\mathrm{a} \mathrm{CaF}_{2}$ window (Crystran Ltd., Dorset, UK). Spectra of collagens were acquired using the point mode. The acquisition time was set to $30 \mathrm{~s} / \mathrm{spectrum}$ and the spectral range to $500-1800 \mathrm{~cm}^{-1}$. For skin analysis, one sample of skin collected at the level of the breast was obtained during cancer breast surgery, from a 41-year-old patient. The skin sample, characterized as normal by standard histology, was frozen, and thin sections $(5 \mu \mathrm{m})$ were deposited on $\mathrm{CaF}_{2}$ windows. Three tissue sections were analyzed by Raman spectroscopy in imaging mode. A total of 6 areas of about $50 \times 50 \mu \mathrm{m}^{2}$ around the DEJ were scanned point by point using a step size of $0.5 \mu \mathrm{m}$.

\section{Results}

\subsection{Raman Analysis of Type I and IV Collagens}

Figure 1 displays typical Raman spectra of type I and IV collagens after baseline correction and normalization on the amide I band. The amide I band is centered at $1668 \mathrm{~cm}^{-1}$ and $1673 \mathrm{~cm}^{-1}$ in the type I and type IV collagens, respectively. They arise from $\mathrm{C}=\mathrm{O}$ stretching of the peptidic bond in the Gly$\mathrm{X}-\mathrm{Y}$ tripeptide sequence. The frequency shift of the amide I band suggests a different proportion of amino acid residues in $\mathrm{X}$ and $\mathrm{Y}$ positions between the two collagens. The amide III region presents also significant differences between the two collagens. The difference of the intensity ratio $\mathrm{I}_{1271} / \mathrm{I}_{1246}$ reflects a difference of proline residues proportion. Indeed, the vibrations at $1246 \mathrm{~cm}^{-1}$ and $1271 \mathrm{~cm}^{-1}$ are assigned to proline-rich and proline-poor regions, respectively [17]. This feature is associated with different spectral profiles in the $820-985 \mathrm{~cm}^{-1}$ range. The peak at $875 \mathrm{~cm}^{-1}$ arises from the hydroxyproline ring [12]. The vibrations at 856 and $920 \mathrm{~cm}^{-1}$ are assigned to the proline ring; the $938 \mathrm{~cm}^{-1}$ band corresponds to the $\mathrm{C}-\mathrm{C}$ stretching vibration $\left(\nu_{\mathrm{C}-\mathrm{C}}\right)$ of the backbone formed by Gly$\mathrm{X}-\mathrm{Y}$ sequences [12]. Considering the normalization of the spectra on amide I band, the content of proline and hydroxyproline residues in collagen IV appears higher than in collagen I. Two bands at 1004 and $1033 \mathrm{~cm}^{-1}$ assigned to phenylalanine are much more intense in collagen IV; this observation could translate a higher content of phenylalanine in collagen IV or conformational differences at the level of these residues between the two proteins. The vibration at $815 \mathrm{~cm}^{-1}$, visible in both collagens and assigned to the $\mathrm{C}-\mathrm{O}-\mathrm{C}$ stretching [18], can be associated with the glucosyl-galactosylhydroxylysinonorleucine cross-link between the tropocollagens [10]. It is more intense in collagen I; this indicates a higher content of cross-links in collagen I than in collagen IV. This spectral feature is correlated with the fibrillar structure of collagen I. Information concerning disulphide bridge can also be extracted from the $500-550 \mathrm{~cm}^{-1}$ spectral range. The S-S vibrations of type IV collagen appear at $510 \mathrm{~cm}^{-1}$ and $540 \mathrm{~cm}^{-1}$. These signals are relative to the disulfide bridges of the $7 \mathrm{~S}$ domains; they indicate that these bridges are in the very stable gauche-gauche-gauche conformation $\left(510 \mathrm{~cm}^{-1}\right)$ and 


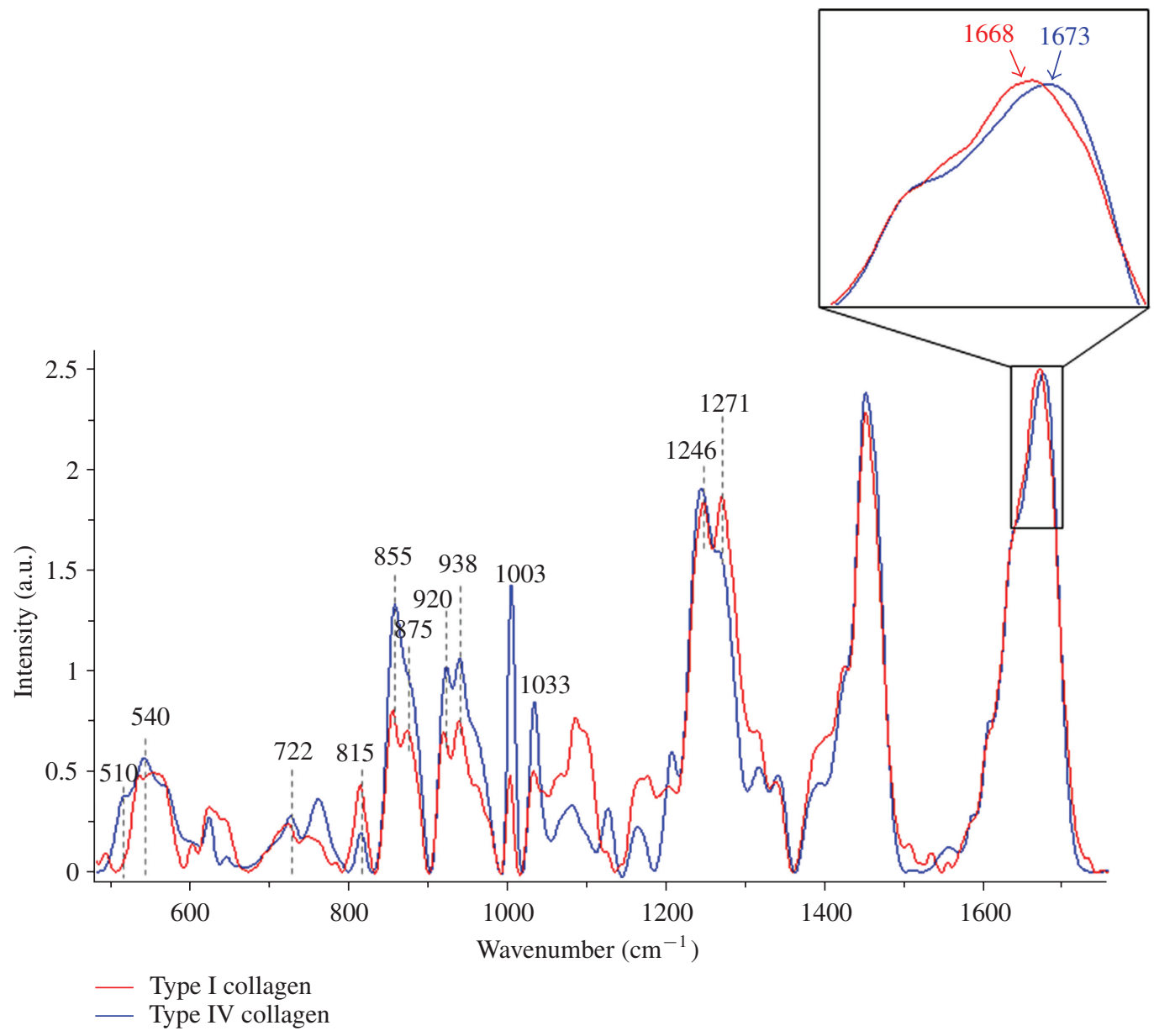

Figure 1: Raman spectra of type I and IV collagens. For comparison, spectra were baseline corrected and normalized on the amide I band.

the less stable trans-gauche-trans conformation $\left(540 \mathrm{~cm}^{-1}\right)$ [19]. These peaks are also associated with the $\mathrm{C}-\mathrm{S}$ stretching vibration at $722 \mathrm{~cm}^{-1}$. This conformational information specific of the $7 \mathrm{~S}$ domain could be used as in situ markers of the stability of the collagen IV macromolecular network. In type I collagen, the $\mathrm{S}-\mathrm{S}$ vibrations at 510 and $540 \mathrm{~cm}^{-2}$ do not appear clearly.

\subsection{Raman Analysis of Epidermis, Dermis, and Epidermis/Dermis Interface}

Figure 2 shows the average and standard deviation spectra $(n=24)$ of epidermis, dermis, and epidermis/dermis interface. Taking into account the signal variability, significant spectral differences between the investigated skin structures are visible at the level of amide I, amide III, and proline bands. The amide I band is centered on the $1668 \mathrm{~cm}^{-1}$ wavenumber in dermis; this value is assigned to collagen I fiber [2]. In the epidermis, the peak at $1654 \mathrm{~cm}^{-1}$ is representative of the $\alpha$-helix structure of keratin [20, 21]. In 


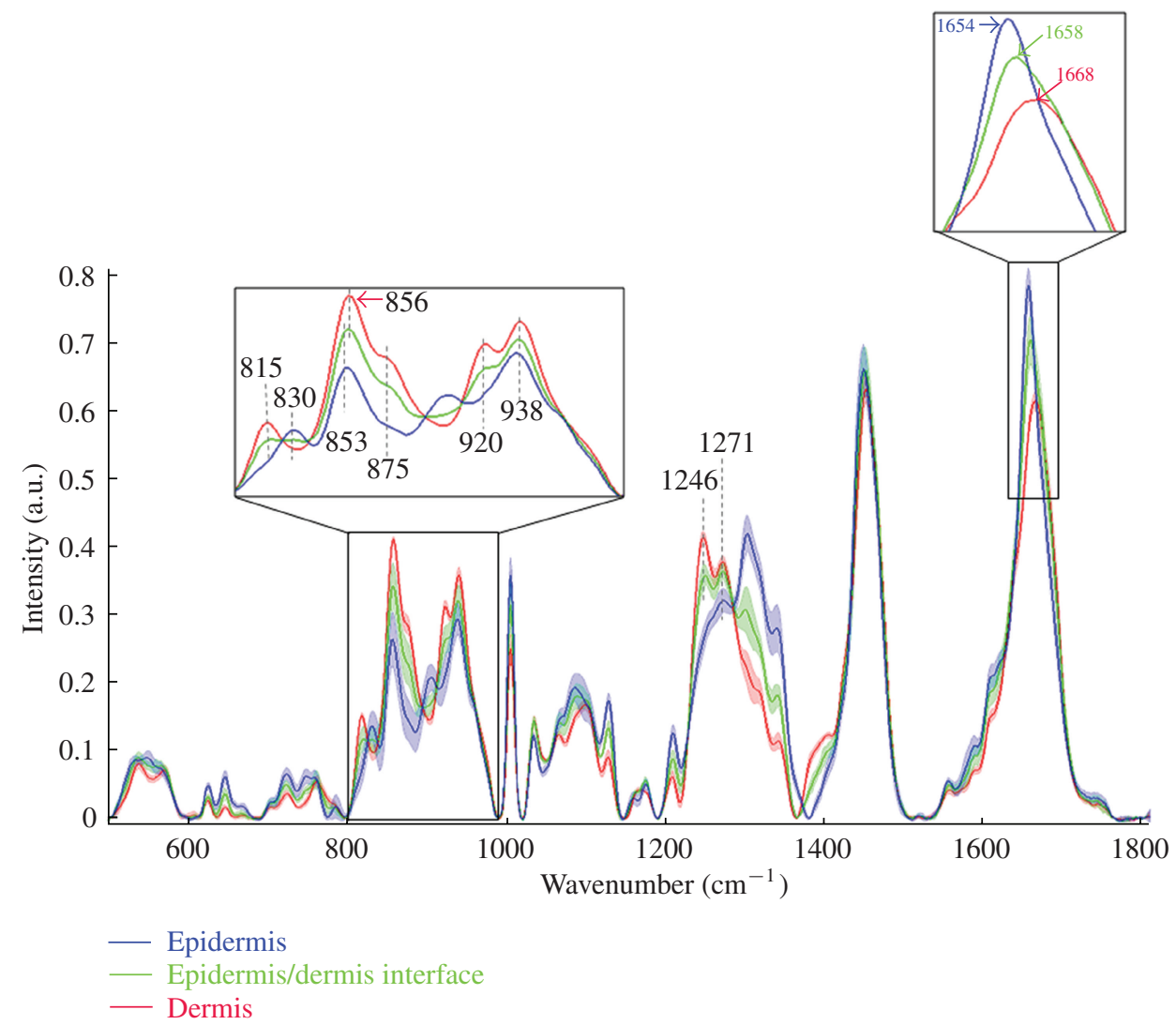

Figure 2: Average spectra and standard deviation of epidermis, dermis, and epidermis/dermis interface. For comparison, spectra were baseline corrected and normalized on the amide I band.

the epidermis/dermis interface, the amide I band is centered on $1658 \mathrm{~cm}^{-1}$, indicating a predominant $\alpha$ helical structure. The structural differences between epidermis, dermis, and epidermis/dermis interface can also be revealed in the amide III region. The vibrations appearing at $1246 \mathrm{~cm}^{-1}$ and $1271 \mathrm{~cm}^{-1}$ are associated with proline-rich (non polar) and proline-poor (polar) regions of the collagens [17]. In the epidermis spectra, the peak at $1271 \mathrm{~cm}^{-1}$ is associated with the $\alpha$-helical structure of keratin [15]. In the $800-980 \mathrm{~cm}^{-1}$ spectral range, the peaks assigned to hydroxyproline $\left(876 \mathrm{~cm}^{-1}\right)$ and proline $\left(920 \mathrm{~cm}^{-1}\right)$ residues present a higher intensity in the spectra of dermis and epidermis/dermis interface than in those of epidermis. The signal at $815 \mathrm{~cm}^{-1}$, assigned to $\mathrm{C}-\mathrm{O}-\mathrm{C}$ stretching vibration of collagens cross-link, is also visible in the dermis and the epidermis/dermis interface but not at the level of the epidermis. The peaks at 830 and $853 \mathrm{~cm}^{-1}$ are assigned to the Fermi doublet of the tyrosyl residues in protein. In the epidermis, the intensity of the peak at $830 \mathrm{~cm}^{-1}$ is higher than in the epidermis/dermis interface. In the dermis this peak is not discernible. In addition, the intensity ratio of the Fermi doublet, informative of the protein conformation at the level of tyrosine residues [22], varies between the skin structures. According to the value of this ratio, we can determine the exposed or buried conformation of tyrosine residues within the protein, inducing either intra- or intermolecular bonding, respectively. In the disulphide bridge spectral range $\left(500-550 \mathrm{~cm}^{-1}\right)$, no spectral difference between epidermis, dermis, 
and epidermis/dermis interface appears clearly. The S-S stretching vibrations specific of collagens are difficult to be discerned because other constituents such as keratin (in epidermis), elastin or fibronectin (in dermis), and laminin (in DEJ) can give superimposed signals.

\section{Discussion and Conclusion}

Our findings show that Raman spectroscopy permits to identify structural differences between the type I and IV collagens. Spectral differences were also clearly detected between the dermis, epidermis and epidermis/dermis interface. Further works will be devoted to in situ analysis on skin section samples. The spectral features specific of type I or IV collagens represent basic data for further studies, particularly to probe skin molecular alterations induced by chronologic aging. In addition, it has to be indicated that spectra collected on the epidermis/dermis interface comprise not only the DEJ signal but also both signals from epidermis and dermis. Indeed, the basement membrane $(\approx 100 \mathrm{~nm})$ of the DEJ is much finer than the nominal spot size of analysis in conventional Raman microspectroscopy. New instrumental developments, based on near-field spectroscopy, are presently carried out to focus the analysis on dermoepidermal junction.

\section{Acknowledgments}

T. T. Nguyen is a recipient of a doctoral fellowship and CIRGE Région Champagne-Ardenne. The authors would like to thank Ligue contre le Cancer (Comité de l'Aisne), INSERM PNR Imagerie, CNRS Projets Exploratoires Pluridisciplinaires, and CNRS PIR Longévité et Vieillissement for financial support.

\section{References}

[1] J. M. Waller and H. I. Maibach, "Age and skin structure and function, a quantitative approach (I): blood flow, $\mathrm{pH}$, thickness, and ultrasound echogenicity," Skin Research and Technology, vol. 11, no. 4, pp. 221-235, 2005.

[2] E. Ly, O. Piot, A. Durlach, P. Bernard, and M. Manfait, "Polarized Raman microspectroscopy can reveal structural changes of peritumoral dermis in basal cell carcinoma," Applied Spectroscopy, vol. 62, no. 10, pp. 1088-1094, 2008.

[3] Y. Maeshima, P. C. Colorado, A. Torre et al., "Distinct antitumor properties' of a type IV collagen domain derived from basement membrane," Journal of Biological Chemistry, vol. 275, no. 28, pp. 21340-21348, 2000.

[4] Y. Maeshima, M. Manfredi, C. Reimerli et al., "Identification of the anti-angiogenic site within vascular basement membrane-derived tumstatin," Journal of Biological Chemistry, vol. 276, no. 18, pp. 15240-15248, 2001.

[5] S. Pasco, J. Han, P. Gillery et al., "A specific sequence of the noncollagenous domain of the $\alpha 3$ (IV) chain of type IV collagen inhibits expression and activation of matrix metalloproteinases by tumor cells," Cancer Research, vol. 60, no. 2, pp. 467-473, 2000.

[6] S. Pasco, L. Ramont, L. Venteo, M. Pluot, F. X. Maquart, and J. C. Monboisse, "In vivo overexpression of tumstatin domains by tumor cells inhibits their invasive properties in a mouse melanoma model," Experimental Cell Research, vol. 301, no. 2, pp. 251-265, 2004. 
[7] N. Floquet, S. Pasco, L. Ramont et al., "The antitumor properties of the $\alpha 3$ (IV)-(185-203) peptide from the NC1 domain of type IV collagen (tumstatin) are conformation-dependent," Journal of Biological Chemistry, vol. 279, no. 3, pp. 2091-2100, 2004.

[8] J. Thevenard, N. Floquet, L. Ramont et al., "Structural and antitumor properties of the YSNSG cyclopeptide derived from tumstatin," Chemistry and Biology, vol. 13, no. 12, pp. 1307-1315, 2006.

[9] F. Vázquez, S. Palacios, N. Alemañ, and F. Guerrero, "Changes of the basement membrane and type IV collagen in human skin during aging," Maturitas, vol. 25, no. 3, pp. 209-215, 1996.

[10] J. P. Borel, "Les collagènes," l Eurobiologiste, vol. 25, no. 195, pp. 247-271, 1991.

[11] K. Beck and B. Brodsky, "Supercoiled protein motifs: the collagen triple-helix and the $\alpha$ - helical coiled coil," Journal of Structural Biology, vol. 122, no. 1-2, pp. 17-29, 1998.

[12] Q. Zhang, K. L. Andrew Chan, G. Zhang et al., "Raman microspectroscopic and dynamic vapor sorption characterization of hydration in collagen and dermal tissue," Biopolymers, vol. 95, no. 9, pp. 607-615, 2011.

[13] R. Kalluri, "Basement membranes: structure, assembly and role in tumour angiogenesis," Nature Reviews Cancer, vol. 3, no. 6, pp. 422-433, 2003.

[14] J. Candiello, G. J. Cole, and W. Halfter, "Age-dependent changes in the structure, composition and biophysical properties of a human basement membrane," Matrix Biology, vol. 29, no. 5, pp. 402-410, 2010.

[15] M. Gniadecka, O. F. Nielsen, S. Wessel, M. Heidenheim, D. H. Christensen, and H. C. Wulf, "Water and protein structure in photoaged and chronically aged skin," Journal of Investigative Dermatology, vol. 111, no. 6, pp. 1129-1132, 1998.

[16] K. A. Piez, E. A. Eigner, and M. S. Lewis, "The chromatographic separation and amino acid composition of the subunits of several collagens," Biochemistry, vol. 2, no. 1, pp. 58-66, 1963.

[17] B. G. Frushour and J. L. Koenig, "Raman scattering of collagen, gelatin, and elastin," Biopolymers, vol. 14, no. 2, pp. 379-391, 1975.

[18] T. Ikoma, H. Kobayashi, J. Tanaka, D. Walsh, and S. Mann, "Physical properties of type I collagen extracted from fish scales of Pagrus major and Oreochromis niloticas," International Journal of Biological Macromolecules, vol. 32, no. 3-5, pp. 199-204, 2003.

[19] M. Gniadecka, O. F. Nielsen, D. H. Christensen, and H. C. Wulf, "Structure of water, proteins, and lipids in intact human skin, hair, and nail," Journal of Investigative Dermatology, vol. 110, no. 4, pp. 393-398, 1998.

[20] A. Kuzuhara, "Analysis of structural changes in bleached keratin fibers (black and white human hair) using Raman spectroscopy," Biopolymers, vol. 81, no. 6, pp. 506-514, 2006.

[21] A. Kuzuhara, N. Fujiwara, and T. Hori, "Analysis of international structure changes in black human hair keratin fibers with aging using Raman spectroscopy," Biopolymers, vol. 87, no. 2-3, pp. 134 140, 2007.

[22] F. S. Parker, Applications of Infrared, Raman, and Resonance Raman Spectroscopy in Biochemistry, Plenum Press, NewYork, NY, USA, 1983. 


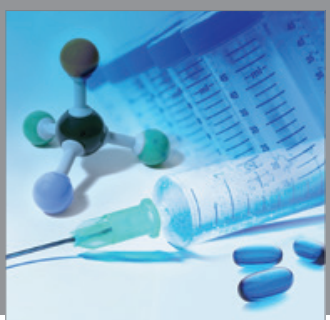

International Journal of

Medicinal Chemistry

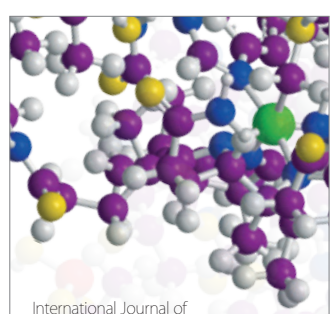

Carbohydrate Chemistry

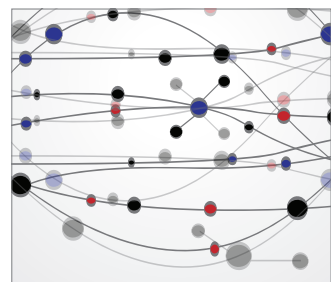

The Scientific World Journal
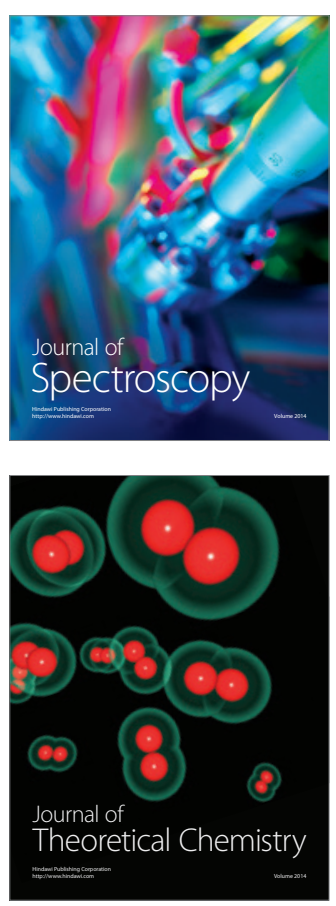
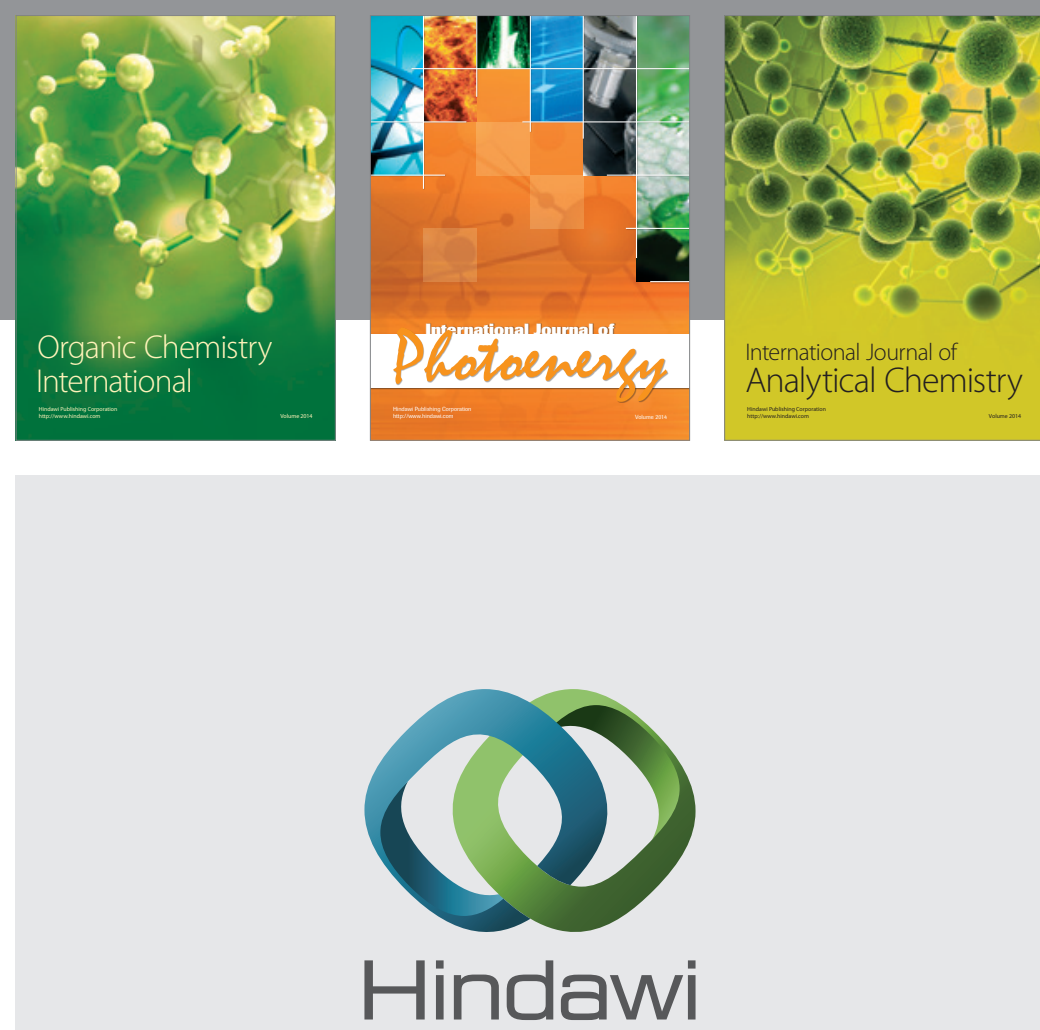

Submit your manuscripts at

http://www.hindawi.com
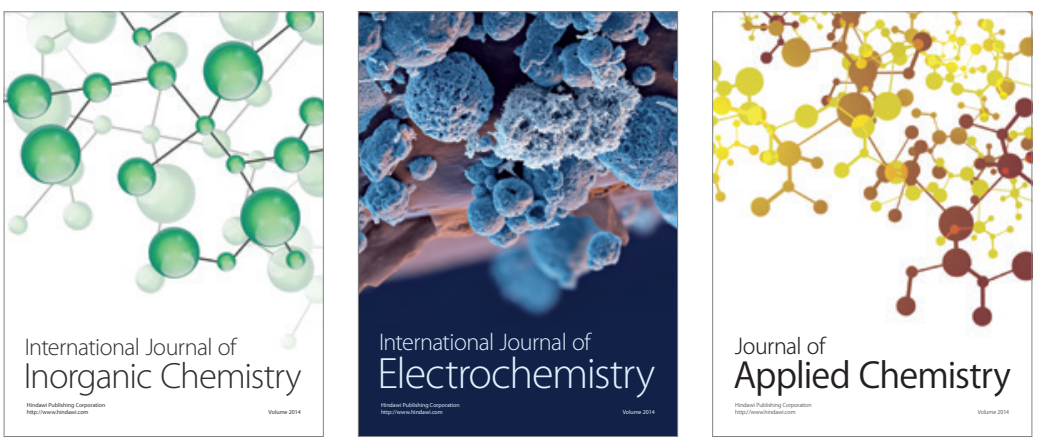

Journal of

Applied Chemistry
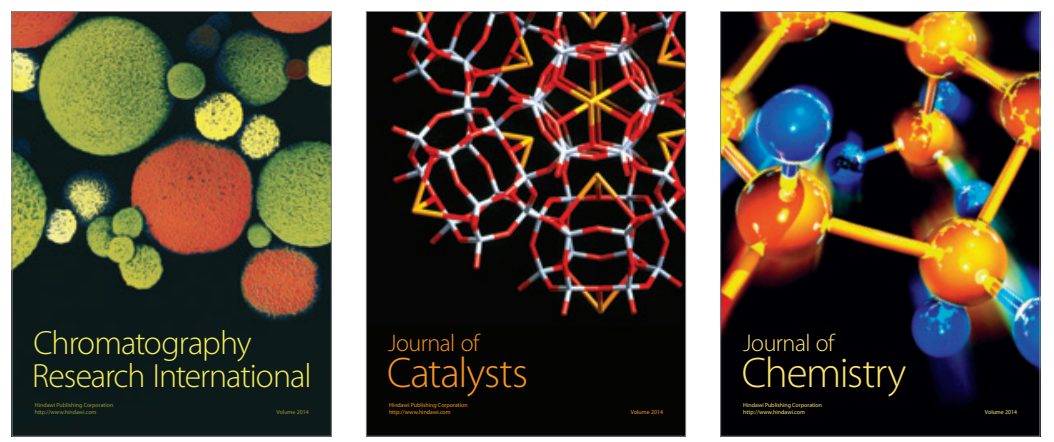
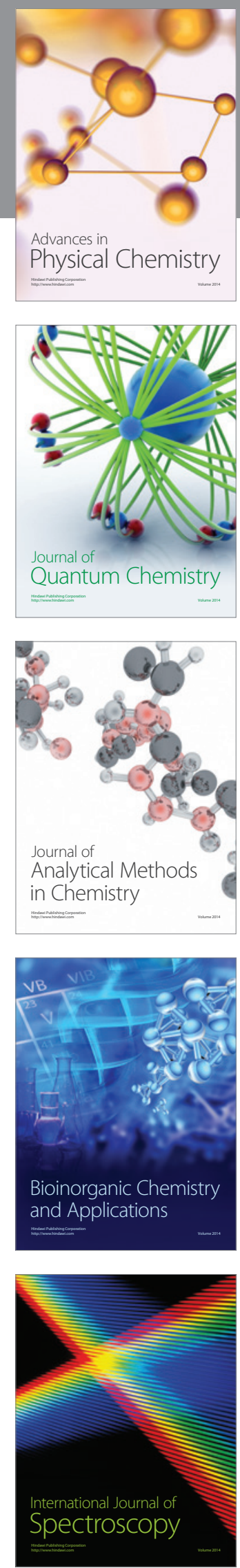